03

\title{
Влияние осаждения и коагуляции частиц на параметры текущих в трубе наноаэрозолей
}

\author{
(C) T.P. Аманбаев \\ Южно-Казахстанский государственный университет им. М. Ауэзова, \\ 160012 Шымкент, Казахстан \\ Институт математики и математического моделирования, \\ 050010 Алматы, Казахстан \\ e-mail: tulegen_amanbaev@mail.ru
}

Поступило в Редакцию 26 апреля 2020 г.

В окончательной редакции 6 сентября 2020 г.

Принято к публикации 25 сентября 2020 г.

\begin{abstract}
Изучено влияние процессов осаждения и коагуляции частиц, обусловленных броуновской диффузией, на параметры аэрозолей, текущих в трубе. Задача рассмотрена в двумерной постановке с учетом неоднородного профиля скорости среды поперек трубы. При разных числах Кнудсена численным методом конечных разностей получены распределения концентрации и радиуса кластеров, сформированных из-за коагуляции частиц, в продольном и поперечном направлениях. Обнаружено, что, двигаясь по каналу, кластеры достигают конечного предельного размера. Обсуждено влияние определяющих параметров на распределение дисперсных характеристик смеси внутри трубы и на предельный размер кластеров.
\end{abstract}

Ключевые слова: броуновская диффузия, диффузионное осаждение, коагуляция, кластер, число Кнудсена.

DOI: $10.21883 /$ JTF.2021.03.50515.146-20

\section{Введение}

Когда мельчайшие (например, наноразмерные) частицы твердого или жидкого вещества движутся вместе с потоком газа через канал, вследствие броуновской диффузии они могут приблизиться к стенкам канала и прилипнуть к ним. Исходя из доли частиц, прошедших через канал, может быть определен броуновский коэффициент диффузии частиц и, следовательно, их размеры. Этот метод широко применяется для определения дисперсности аэрозолей и используется на практике достаточно давно [1-7]. Кроме того, процесс диффузионного осаждения частиц используется также при тонкой очистке от вредных аэрозольных частиц небольших объемов газа (в частности, при создании стерильных условий в медицинской и микробиологической промышленности, точном машиностроении и т.п.) [8]. Изучение течений аэрозолей в каналах имеет важное значение в медицинских приложениях (движение аэрозолей в дыхательных путях и т.п.) [9]. В последнее время возникла необходимость более детального исследования течений наноаэрозолей в связи с бурным развитием наноаэрозольных технологий, таких, как получение нанопорошков и наноаэрозолей с заданными характеристиками, нанесение заданной толщины специальных покрытий из наночастиц, получение высококачественных оптических волокон и т.п. $[10,11]$.

Течение аэрозолей зачастую может сопровождаться коагуляцией частиц [3,12-17], что приводит к росту размеров частиц, и, как следствие, сказывается на их подвижности. Оценки показывают, что при достаточной концентрации частиц процесс коагуляции сильно влияет на размеры кластеров и его надлежит учитывать при анализе дисперсного состава аэрозолей, текущих в каналах. Между тем совместное влияние процессов броуновского осаждения и коагуляции на параметры дисперсной смеси, текущей в трубе, практически не изучено. Отметим работу [13], где наряду с осаждением и коагуляцией учитывается еще термофорез, связанный с наличием градиента температуры газа. При этом изменение размеров частиц, обусловленное их коагуляцией, в [13] не анализируется.

Цель настоящей работы - изучение влияния процессов диффузионного осаждения частиц на стенки трубы и их коагуляции на дисперсные характеристики потока аэрозолей в трубе.

В настоящей работе под термином „частица“ подразумевается как собственно твердая частица, так и жидкая капля. Наряду с термином „частица“ применительно к твердым частицам используется также термин „кластер“.

\section{1. Основные допущения и постановка задачи}

\section{1. Основные допущения}

Рассматривается стационарное движение смеси газа с мелкими (радиусом $\lesssim 100 \mathrm{~nm})$ частицами в трубе. Полагается, что процессы коагуляции частиц и их осаждения на стенки канала происходят за счет броуновской диффузии. В качестве характерного размера кластера, появляющегося из-за коагуляции частиц, берется радиус шара, объем которого равен объему кластера. Считается, что смесь локально-монодисперсна (в каждом 
элементарном объеме имеются частицы только одного размера). Радиус трубы намного больше средней длины свободного пробега молекулы газа и радиуса частиц, но значительно меньше длины трубы.

Течение в трубе происходит при небольших числах Рейнольдса, так что течение среды можно считать ламинарным с параболическим профилем скорости поперек трубы. Оценки показали, что влиянием поперечной силы Сэфмана, появляющейся в таких сдвиговых потоках, в рассматриваемых условиях можно пренебречь. Полагается, что межфазное скольжение отсутствует. Данное предположение обосновывается тем, что при радиусах частиц 1-100 nm характерное время выравнивания скоростей фаз в стоксовом режиме с учетом поправки Каннингема-Милликена (учитывающего изменение условий обтекания малых частиц) [12] составляет порядка $10^{-8}-10^{-6} \mathrm{~s}$, что намного меньше времени пребывания смеси в трубе. В предельном случае отсутствия межфазного скольжения поперечная сила в сдвиговых потоках равна нулю независимо от профиля скорости и наличия стенок [18]. Процесс гравитационной седиментации дисперсных частиц из-за малости их размеров не учитывается. Задача рассматривается в двумерной постановке с осевой симметрией. Диффузией частиц в осевом направлении трубы, ввиду преобладания в этом направлении конвективного движения, пренебрегается.

\section{2. Постановка задачи}

Уравнения сохранения числа частиц и их объемного содержания для двумерного, с осевой симметрией, стационарного движения аэрозолей в цилиндрической системе координат в рамках принятых допущений имеют вид (уравнения конвективной диффузии):

$$
\begin{gathered}
2 U\left(1-\frac{r^{2}}{R^{2}}\right) \frac{\partial n}{\partial x}=\frac{1}{r} \frac{\partial}{\partial r}\left(r D \frac{\partial n}{\partial r}\right)-J, \\
2 U\left(1-\frac{r^{2}}{R^{2}}\right) \frac{\partial \alpha}{\partial x}=\frac{1}{r} \frac{\partial}{\partial r}\left(r D \frac{\partial \alpha}{\partial r}\right), \\
\alpha=\alpha^{0} n, \quad \alpha^{0}=\frac{4}{3} \pi a^{3},
\end{gathered}
$$

где $n, \alpha-$ концентрация частиц и их объемная доля, которые связаны между собой соотношениями (3), $a, \alpha^{0}-$ радиус и объем частицы (кластера), $x, r$ - продольная и поперечная координаты, $R$ - радиус трубы, $D-$ коэффициент диффузии частиц, $J-$ интенсивность коагуляции частиц. Множитель $2 U\left(1-r^{2} / R^{2}\right)$ перед производными по $x$ представляет пуазейловский (параболический) профиль скорости ( $U-$ средняя по поперечному сечению скорость). Следует отметить, что в уравнении для объемной доли частиц (2) отсутствует член, ответственный за коагуляцию, так как очевидно, что коагуляция частиц никак не влияет на объемное содержание дисперсного компонента. При известных значениях $n$ и $\alpha$ соотношения (3) позволяют находить радиус кластеров.
В случае отсутствия коагуляции $(J=0)$ уравнения (1) и (2) оказываются эквивалентными. В уравнениях (1), (2) коэффициент диффузии $D$ зависит, вообще говоря, от радиуса кластеров $a$, а также от других параметров, см. ниже. В общем случае, в частности, при наличии коагуляции величины $a$, а потому и $D$ не постоянны во всем объеме смеси, так что параметр $D$ не может быть вынесен из-под знака производной.

Поставим граничные условия: на оси трубы примем условие симметрии течения, на стенках канала - условие равенства нулю концентрации и объемного содержания частиц (условие исчезновения частиц), а на границе $x=0$ примем условия во входном сечении трубы:

$$
\begin{aligned}
\frac{\partial n}{\partial r}(0, x) & =0, \quad \frac{\partial \alpha}{\partial r}(0, x)=0, \\
n(R, x)=0, \quad \alpha(R, x) & =0, \quad n(r, 0)=n_{0}, \quad \alpha(r, 0)=\alpha_{0} .
\end{aligned}
$$

Здесь $n_{0}, \alpha_{0}$ - концентрация и объемная доля частиц во входном сечении трубы.

Учитывая принятые допущения о монодисперсности смеси и о том, что коагуляция частиц происходит в результате их броуновской диффузии, выражение для $J$ в первом приближении запишем в форме $[3,11,12]$ :

$$
J=\frac{K}{2} n^{2}
$$

где $K$ - так называемая константа (или коэффициент) коагуляции.

\section{3. Коэффициенты дифффузии и коагуляции}

Для коэффициента диффузии $D$ имеем формулу Эйнштейна (с поправочным коэффициентом $C$ ) [12]:

$$
D=\frac{k_{B} T}{6 \pi \mu a} C,
$$

где $k_{B}$ - постоянная Больцмана, $\mu, T-$ вязкость и температура несущей среды. Поправочный коэффициент $C$ учитывает переход от сплошного режима течения вокруг частицы к свободно-молекулярному и зависит от числа Кнудсена $\mathrm{Kn}$, увеличиваясь с ростом Kn. Для $C$ имеется следующее выражение, найденное в [19] теоретически (на основе приближенного решения уравнения Больцмана):

$$
\begin{gathered}
C=\frac{15+12 c_{1} \mathrm{Kn}+9\left(c_{1}^{2}+1\right) \mathrm{Kn}^{2}+18 c_{2}\left(c_{1}^{2}+2\right) \mathrm{Kn}^{3}}{15-3 c_{1} \mathrm{Kn}+c_{2}(8+\pi \sigma)\left(c_{1}^{2}+2\right) \mathrm{Kn}^{2}}, \\
c_{1}=\frac{2-\sigma}{\sigma}, \quad c_{2}=\frac{1}{2-\sigma}, \quad \mathrm{Kn}=\frac{\lambda}{a} .
\end{gathered}
$$

Здесь $\lambda-$ средняя длина свободного пробега молекулы газа. Параметр $\sigma$ принимает значения от 0.79 до 1.0 [19]. Данная формула описывает величину С во всем диапазоне изменения числа $\mathrm{Kn}$, включая как предельные ситуации малых и больших значений $\mathrm{Kn}$, соответствующие сплошному $(\mathrm{Kn} \ll 1)$ и свободно-молекулярному 
$(\mathrm{Kn} \gg 1)$ режимам, так и промежуточных значений числа $\mathrm{Kn}(\mathrm{Kn} \sim 1)$. Необходимо отметить, что представленное выше соотношение для $C$ хорошо согласуется с поправочным коэффициентом в форме Каннингема-Милликена [20]. Следуя [20], положим $\sigma=1$, тогда формула для $C$ примет более простой вид

$$
C=\frac{5+4 \mathrm{Kn}+6 \mathrm{Kn}^{2}+18 \mathrm{Kn}^{3}}{5-\mathrm{Kn}+(8+\pi) \mathrm{Kn}^{2}} .
$$

В таком виде поправочный коэффициент $C$ используется в [14]. Заметим, что имеются также и другие соотношения для $C[21,22]$, которые примерно одинаково описывают зависимость поправочного коэффициента $C$ от числа Кнудсена.

Коэффициент коагуляции зависит от режима обтекания частицы, и в предельных случаях сплошного и свободно-молекулярного режимов выражается известными формулами $[13,20]$. В промежуточном режиме можно воспользоваться известным подходом Фукса, приводящим к приближенной зависимости коэффициента коагуляции от размера частиц и других параметров дисперсной смеси - вязкости и температуры газа, плотности частиц и т.п. [3], которая часто применяется для исследования процесса коагуляции в дисперсных системах $[14,23]$

$$
\begin{gathered}
K=16 \pi a D\left(\frac{a}{2 a+\sqrt{2} g}+\frac{\sqrt{2} D}{c a}\right)^{-1}, \\
g=\frac{1}{6 a l}\left[(2 a+l)^{3}-\left(4 a^{2}+l^{2}\right)^{3 / 2}\right]-2 a, \\
l=\frac{8 D}{\pi c}, \quad c=\left(\frac{8 k_{B} T}{\pi \rho_{p}^{0} \alpha^{0}}\right)^{1 / 2}, \quad \alpha^{0}=\frac{4}{3} \pi a^{3} .
\end{gathered}
$$

Здесь $l, c-$ „кажущаяся““ средняя длина свободного пути частиц и их средняя скорость [3], $\rho_{p}^{0}-$ плотность вещества частиц. Как отмечено в [23], имеется достаточное количество экспериментальных и теоретических исследований по измерению и расчету коэффициента коагуляции в переходном режиме [24-27], результаты которых удовлетворительно согласуются с формулой (8).

Таким образом, уравнения (1)-(3) с граничными условиями (4) и соотношения (5)-(8) составляют полную систему, описывающую поведение дисперсных параметров аэрозолей, движущихся в трубе при наличии процессов осаждения и коагуляции частиц.

\section{2. Безразмерные переменные и определяющие параметры}

Введем безразмерные переменные:

$$
\begin{gathered}
\xi=\frac{x D_{\lambda}}{2 U R^{2}}, \quad \bar{r}=\frac{r}{R}, \quad \bar{n}=\frac{n}{n_{0}}, \quad \bar{\alpha}=\frac{\alpha}{\alpha_{0}}, \\
\bar{a}=\frac{a}{a_{0}}=\left(\frac{\bar{\alpha}}{\bar{n}}\right)^{1 / 3}, \quad D_{\lambda}=\frac{k_{B} T}{6 \pi \mu \lambda} .
\end{gathered}
$$

Параметр $D_{\lambda}$ можно трактовать как коэффициент диффузии частицы, радиус которой равен средней длине свободного пробега молекулы окружающей газовой среды.

Анализ безразмерных уравнений показал, что дисперсный состав движущихся в трубе аэрозолей при наличии процессов осаждения и коагуляции частиц определяется тремя безразмерными критериями:

$$
\mathrm{Kn}_{0}=\frac{\lambda}{a_{0}}, \quad \Phi=\pi R^{2} \lambda n_{0}, \quad B=\mu\left(\frac{\lambda}{k_{B} T \rho_{p}^{0}}\right)^{1 / 2}
$$

Параметр $\mathrm{Kn}_{0}$ при фиксированном $\lambda$ фактически устанавливает начальный размер частиц (критерий Кнудсена часто применяют для классификации частиц по размерам [10]). Параметр Ф представляет собой число частиц в цилиндрической трубе с радиусом основания $R$ и высотой $\lambda$ и отвечает за интенсивность процесса коагуляции. При фиксированных $\lambda$ и $R$ задание значения $\Phi$ равносильно заданию начальной концентрации частиц $n_{0}$. Оценки показали, что для смеси воздуха с характерными значениями физико-химических параметров $\mu \sim 10^{-5} \mathrm{~kg} /(\mathrm{m} \cdot \mathrm{s}), \lambda \sim 0.1 \mu \mathrm{m}, T \sim 10^{2} \mathrm{~K}$, $k_{B} \sim 10^{-23} \mathrm{~J} / \mathrm{K}$ и частиц плотностью $\rho_{p}^{0} \sim 10^{3} \mathrm{~kg} / \mathrm{m}^{3}$ критерий $B \sim 1$.

Важно отметить, что при отсутствии коагуляции течение смеси в трубе в рамках принятого здесь способа обезразмеривания характеризуется единственным критерием - числом Кнудсена $\mathrm{Kn}_{0}$.

Таким образом, основными определяющими параметрами поставленной задачи являются два параметра $\mathrm{Kn}_{0}$ и $\Phi$, которые характеризуются при фиксированных $\lambda$ и $R$ соответственно начальным радиусом частиц и их концентрацией.

Далее для удобства верхнюю черту у безразмерных переменных опускаем.

\section{3. Осредненные по поперечному сечению трубы характеристики потока}

Одними из важных характеристик потока смеси являются осредненные по поперечному сечению трубы концентрация частиц $N$, их объемная доля А и радиус $A$ (здесь и далее следует отличать латинскую $A$ от греческой А). Средняя концентрация определяется из условия равенства расходов числа частиц:

$$
\int_{0}^{1} 2 \pi r u(r, \xi) n(r, \xi) d r=N(\xi) \int_{0}^{1} 2 \pi r u(r, \xi) d r
$$

где $u$ - безразмерная (отнесенная к средней скорости $U$ ) скорость потока в трубе, которая в рассматриваемом здесь случае равна $u=2\left(1-r^{2}\right)$ (пуазейловский 
профиль скорости). Таким образом, для средней по сечению трубы концентрации имеем:

$$
N(\xi)=\frac{\int_{0}^{1} r\left(1-r^{2}\right) n(r, \xi) d r}{\int_{0}^{1} r\left(1-r^{2}\right) d r}=4 \int_{0}^{1} r\left(1-r^{2}\right) n(r, \xi) d r .
$$

Аналогично определяется средняя по поперечному сечению объемная доля частиц:

$$
\mathrm{A}(\xi)=\frac{\int_{0}^{1} r\left(1-r^{2}\right) \alpha(r, \xi) d r}{\int_{0}^{1} r\left(1-r^{2}\right) d r}=4 \int_{0}^{1} r\left(1-r^{2}\right) \alpha(r, \xi) d r
$$

Средний радиус частиц по поперечному сечению рассчитывается по формуле

$$
A=\left(\frac{\mathrm{A}}{N}\right)^{1 / 3} .
$$

Далее под терминами „средняя концентрация“ и „средний радиус“ будем подразумевать осредненные по сечению трубы значения концентрации и радиуса кластеров соответственно.

\section{4. Результаты расчетов}

Ниже обсуждаются некоторые результаты расчетов, полученные численным интегрированием конечноразностным методом [28] системы дифференциальных уравнений (1), (2) с граничными условиями (4) (после раскрытия производной по радиальной координате и приведения системы к безразмерной форме). Размеры шагов по осевой и радиальной координатам выбирались такими, чтобы выполнялся устойчивый счет.

Распределение вдоль трубы средней концентрации $N$ при отсутствии коагуляции, полученное здесь расчетным путем, сравнивалось с известным приближенно- аналитическим решением Гормлея-Кеннеди [2]. Сравнение показало почти полное их совпадение, что подтверждает корректность счета конечно-разностным методом.

Имеет смысл сравнить результаты вычислений с расчетными данными, приведенными в [13], где наряду с осаждением и коагуляцией учитывается еще термофорез в сильно нагретой смеси кислорода с кремниевыми частицами в трубе, с пуазейловским распределением скорости аэрозолей. Из-за отрицательного поперечного градиента температуры термофорез приводил к дополнительному перемещению частиц в сторону стенки канала и тем самым усиливал эффект осаждения частиц на стенки канала. Для коэффициента коагуляции в промежуточном режиме (когда $\left.\mathrm{Kn}_{0} \sim 1\right)$ в [13] используется гармоническое среднее между значениями коэффициента в предельных сплошном и свободно-молекулярном

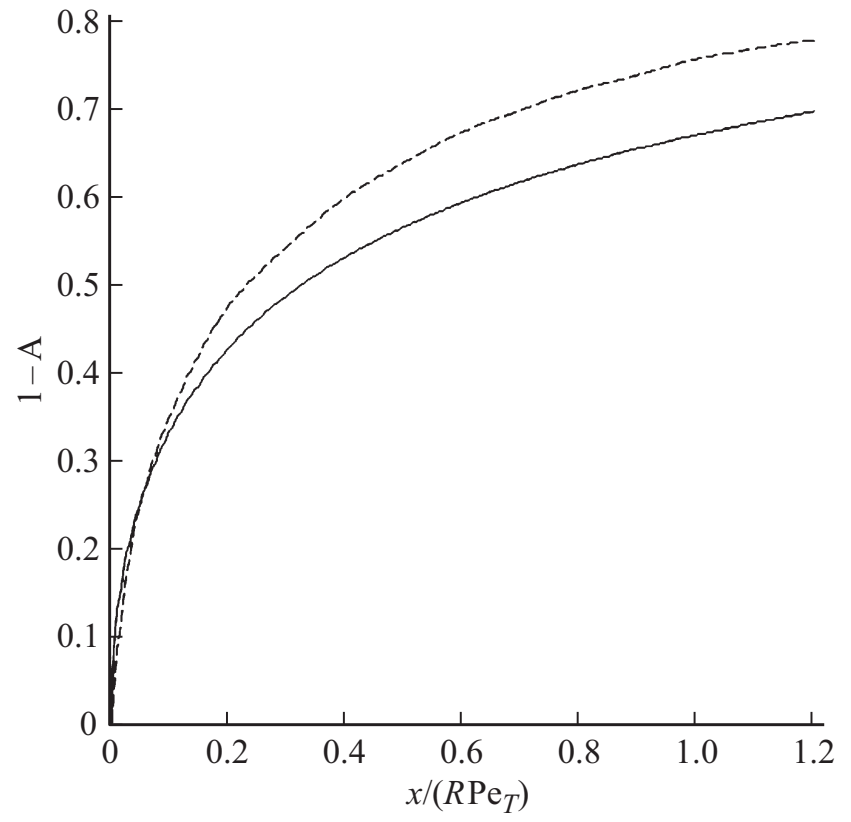

Рис. 1. Эффективность осаждения частиц диаметром $1 \mathrm{~nm}$ и концентрацией $n_{0}=10^{16} 1 / \mathrm{m}^{3}$ вдоль трубы радиусом $0.01 \mathrm{~m}$ : сплошная кривая - результаты расчетов, полученные в настоящей работе, штриховая - данные [13] $\left(\mathrm{Pe}_{T}\right.$ - температурное число Пекле, равно 42.6).

режимах. На рис. 1 сравниваются результаты расчетов эффективности осаждения $(1-\mathrm{A})$ частиц, полученные в настоящей работе и в [13]. Сравнение показывает, что они отличаются друг от друга не очень сильно, причем величина эффективности осаждения из [13] в подавляющей части интервала изменения безразмерной координаты несколько превышает данные настоящей работы, что объясняется, в частности, влиянием термофореза частиц, учитываемого в [13].

Далее проанализируем результаты расчетов, полученные при различных значениях основных определяющих параметров. На рис. 2, 3 продемонстрированы характеры изменения средних по поперечному сечению концентрации $N$ (рис. $2, a, 3, a$ ) и размера кластеров $A$ (рис. $2, b, 3, b)$ вдоль трубы для фиксированных значений числа Кнудсена $\mathrm{Kn}_{0}=1 ; 10$ (в частности, для воздуха при нормальных условиях эти значения соответствуют радиусам частиц 70 и $7 \mathrm{~nm}$ ) и разных значений параметра Ф. Для сравнения штриховыми кривыми показаны распределения параметров при отсутствии коагуляции. Видно, что при наличии коагуляции концентрация частиц уменьшается быстрее, чем при его отсутствии (за исключением небольшой зоны, соответствующей малым $N \ll 1)$. С ростом параметра $\Phi$ средняя концентрация частиц уменьшается, что связано с усилением влияния процесса коагуляции.

По мере движения смеси вдоль трубы кластеры достигают некоторого предельного размера $A_{*}$ на определенном расстоянии от входа $\xi_{*}$. Связано это с тем, что 

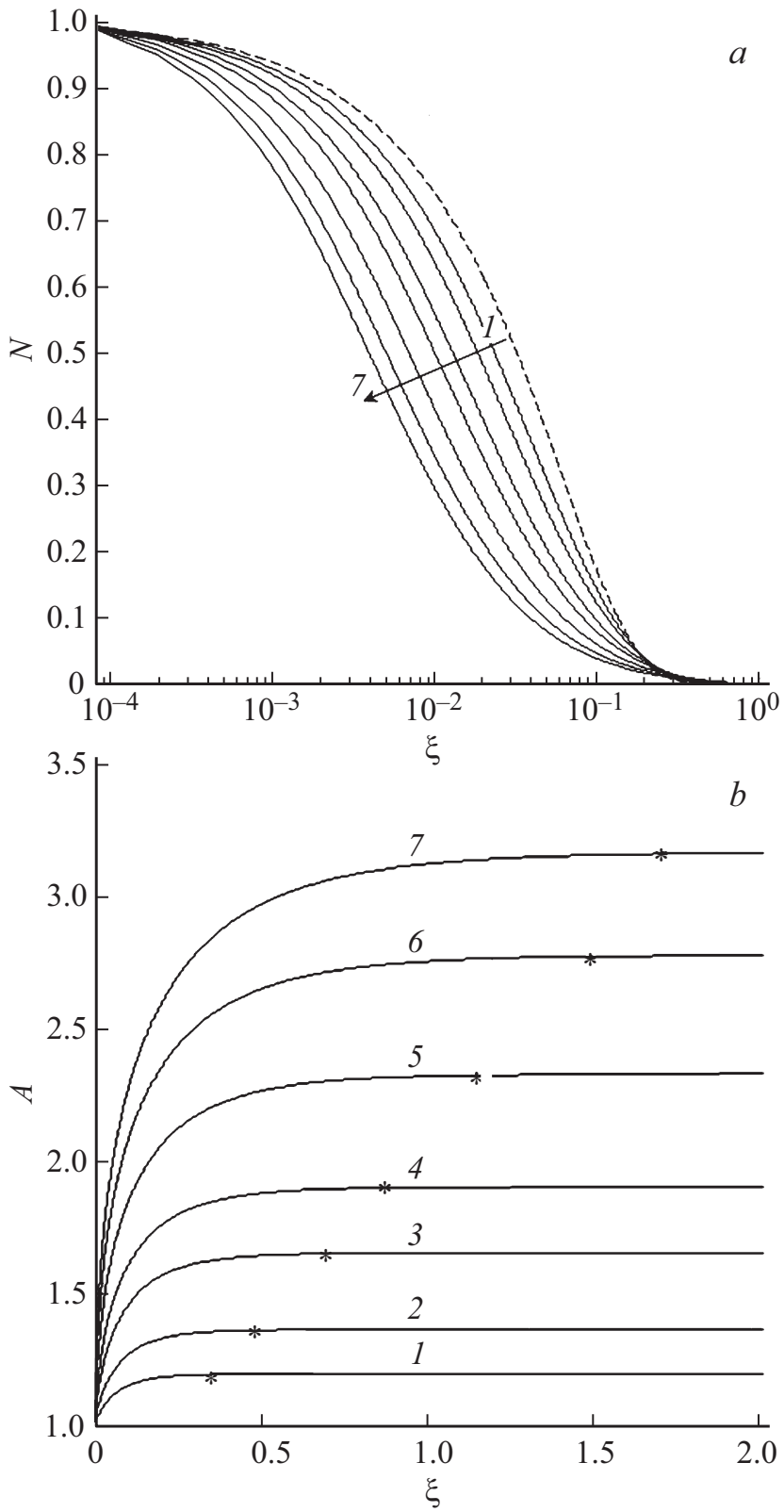

Рис. 2. Распределения безразмерных осредненных по сечению трубы концентрации $(a)$ и радиуса $(b)$ кластеров вдоль трубы при $\mathrm{Kn}_{0}=1$ и разных значениях параметра $\Phi=0.5$ (кривая 1 ), 1 (2), 2 (3), 3 (4), 5 (5), 7.5 (6), 10 (7). Штриховая кривая отсутствие коагуляции.

процесс осаждения частиц лимитирует рост кластеров из-за коагуляции, уменьшая их концентрацию и объем в смеси, причем увеличение параметра $\Phi$ приводит к росту предельного размера кластеров. Для наглядности символом „*๘ на графиках показано приблизительное положение характерной точки $\xi_{*}$, где кластер достигает предельного размера $A_{*}$. Видно, что расстояние $\xi_{*}$ с ростом $\Phi$ существенно увеличивается.

Сравнивая рис. 2, 3 между собой, можно отметить, что с увеличением числа Кнудсена эффект коагуляции проявляется при бо́льших значениях $Ф$, при этом про- цессы осаждения и коагуляции происходят значительно быстрее (об этом, в частности, свидетельствует заметное сокращение характерного расстояния $\xi_{*}$ ). Последнее вполне объяснимо, так как с ростом числа Кнудсена увеличивается коэффициент диффузии, который ответственен за интенсивность процессов осаждения и коагуляции.

На рис. 4 показаны характерные распределения безразмерной концентрации частиц в радиальном направлении в разных поперечных сечениях канала. Для сравнения штриховыми кривыми представлены распределения концентрации при отсутствии процесса коагуляции. Видно, что по мере отдаления от входа неоднород-
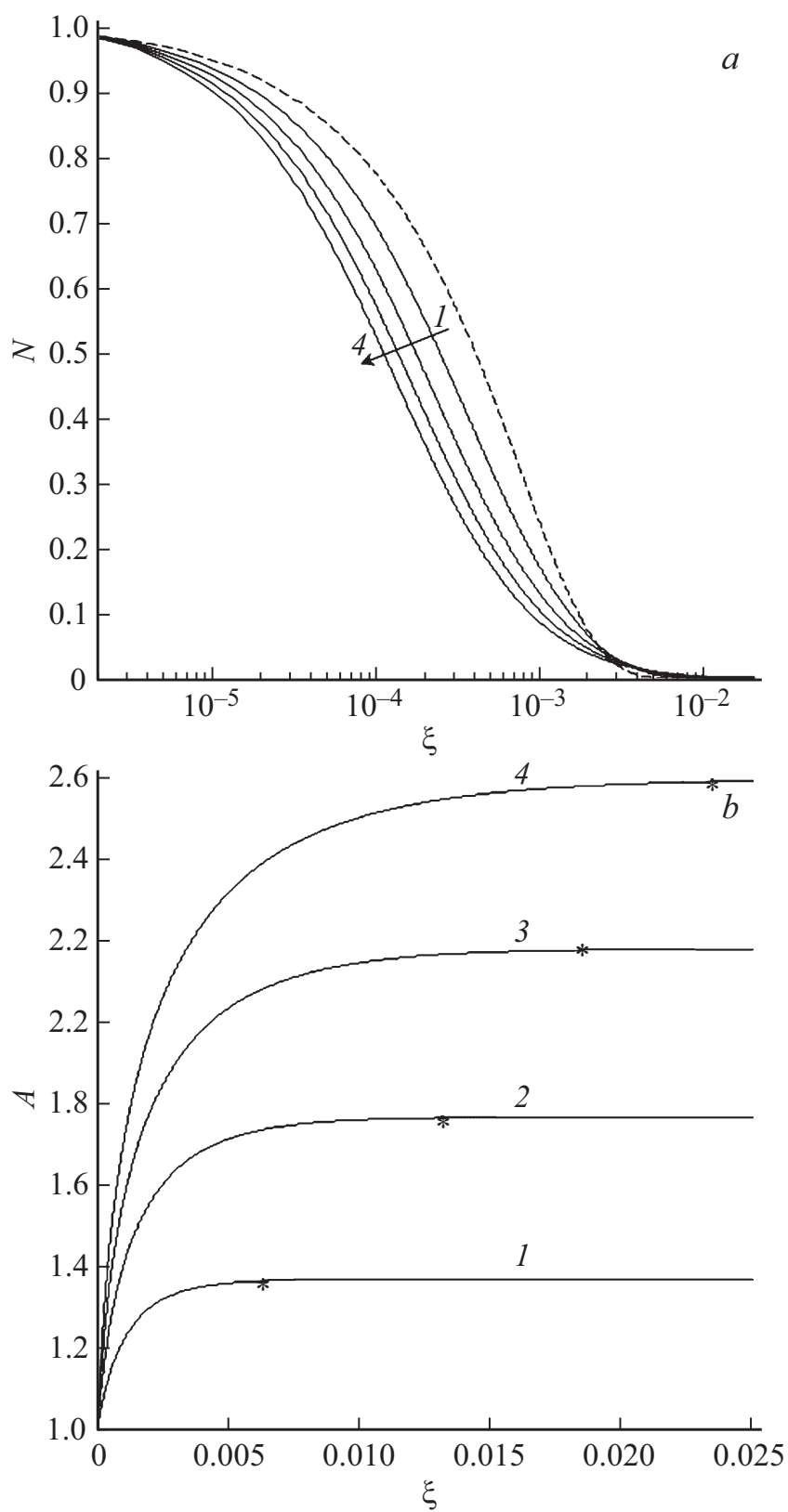

Рис. 3. То же, что на рис. 2 , но при $\mathrm{Kn}_{0}=10$ : $\Phi=50$ (кривая 1), 100 (2), 150 (3), 200 (4). Штриховая кривая отсутствие коагуляции. 
ность распределения концентрации постепенно спадает. Концентрация частиц в сечениях недалеко от входа при наличии коагуляции меньше, чем при его отсутствии, что объясняется уменьшением концентрации из-за коагуляции. Однако по мере удаления от входа трубы различие между распределениями концентрации при наличии и в отсутствие коагуляции, постепенно уменьшаясь, исчезает, и далее наступает ситуация, когда концентрация частиц при наличии коагуляции становится даже больше, чем при ее отсутствии (кривые 5,6, отвечающие распределениям концентрации в сечениях с безразмерными координатами $\xi=0.2$ и 0.3 ). Такое поведение концентрации обусловлено уменьшением подвижности кластеров в броуновском движении из-за их укрупнения за счет коагуляции и наглядно демонстрирует влияние изменения размеров кластеров при коагуляции на интенсивность броуновской диффузии. Этим же обстоятельством объясняется отмеченная выше особенность поведения средней концентрации $N$ при наличии и в отсутствие коагуляции в области малых значений $N$ (рис. $2, a, 3, a$ ), где кривые распределения $N$, несмотря на наличие коагуляции (сплошные кривые), проходят выше, чем в случае ее отсутствия (штриховая линия).

Расчеты показали, что распределения размеров кластеров в радиальном направлении практически однородны, так что радиус кластеров можно считать функцией, зависящей только от одной переменной, а именно от продольной координаты $\xi$. Это означает, что коэффициент броуновской диффузии $D$ в уравнениях (1), (2) вполне можно выносить за знак производной по $r$. При этом уравнение (2) можно решить с помощью

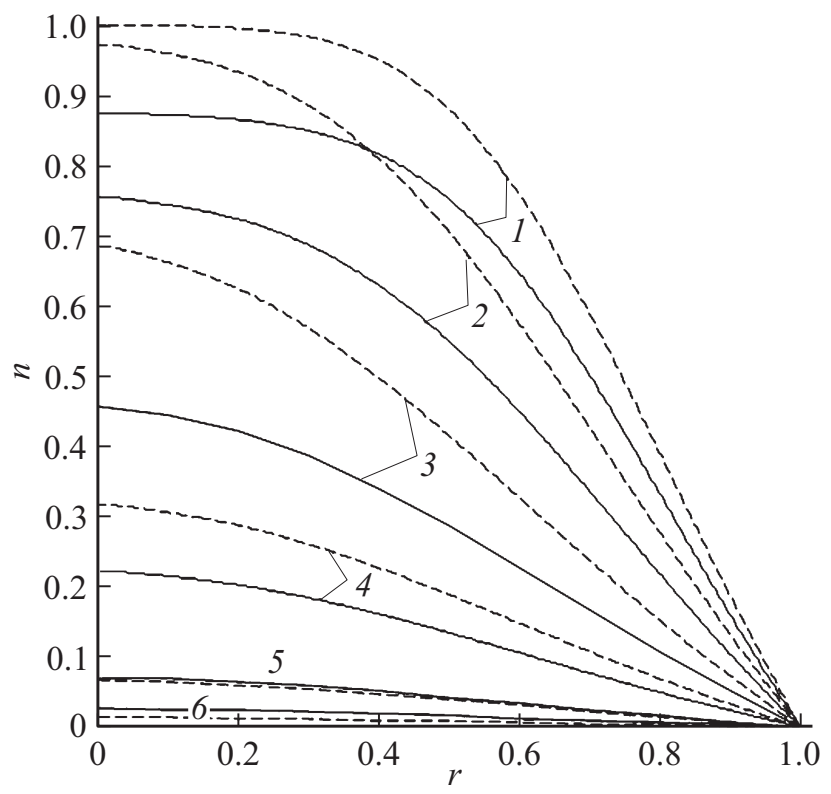

Рис. 4. Распределения безразмерной концентрации частиц в радиальном направлении на разных поперечных сечениях при $\mathrm{Kn}_{0}=1, \Phi=1: 1-\xi=0.01,2-0.02,3-0.05,4-0.1$, $5-0.2,6-0.3$. Штриховая кривая - отсутствие коагуляции.

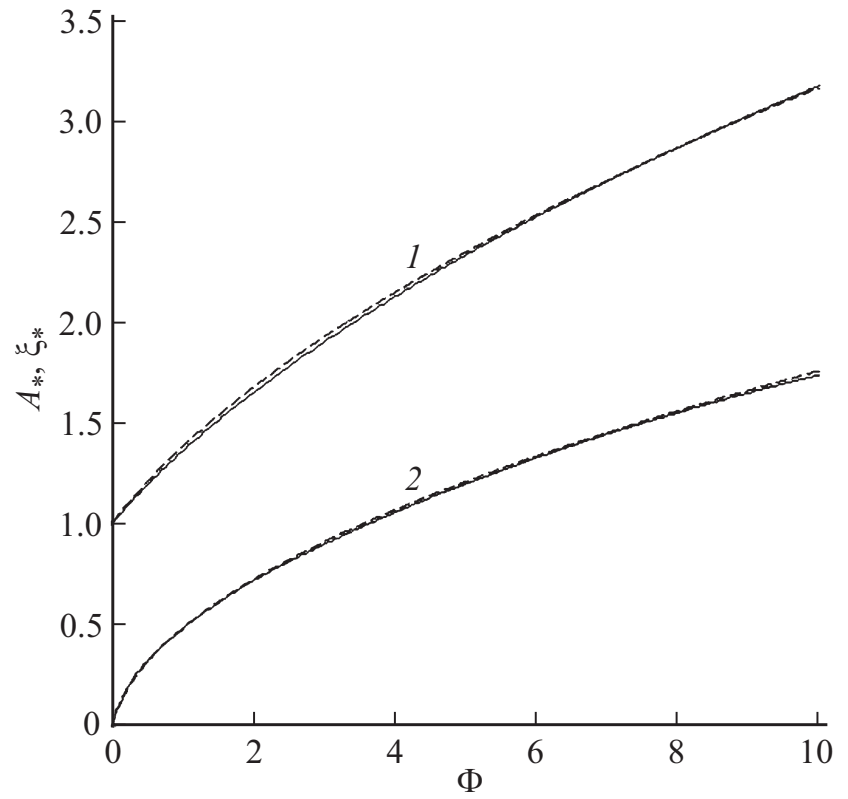

Рис. 5. Зависимости предельного радиуса $A_{*}$ (кривая 1) и характерной координаты $\xi_{*}(2)$ от параметра $\Phi$ при $\mathrm{Kn}_{0}=1$. Штриховые кривые - аппроксимационные формулы (9).

подхода, примененного в [2] для уравнения (1) при отсутствии коагуляции $(J=0)$, и получить приближенно-аналитическое решение отдельно для $\alpha$ (аналогичное установленному в [2] решению для $n)$.

Зависимости предельного радиуса кластеров $A_{*}$ и характерной безразмерной координаты $\xi_{*}$ от параметра $\Phi$ при числе Кнудсена $\mathrm{Kn}_{0}=1$ подробно проиллюстрированы на рис. 5. Для небольших значений $\Phi$ величина $\xi_{*}$ с ростом $\Phi$ резко увеличивается, затем растет плавно. Полученные расчетные зависимости величин $A_{*}$ и $\xi_{*}$ от $\Phi$ достаточно хорошо аппроксимируются следующими функциями:

$$
A_{*}=\sqrt{1+\frac{\pi}{3.5} \Phi}, \quad \xi_{*}=0.13(\sqrt{1+21 \Phi}-1),
$$

графики которых на рис. 5 показаны штриховыми кривыми.

\section{Заключение}

Построена замкнутая система уравнений, описывающая изменение параметров дисперсной смеси (включая размеры кластеров), движущейся в трубе при наличии процессов осаждения и коагуляции частиц. Установлены основные определяющие критерии рассматриваемой задачи. Расчетным путем получены распределения концентрации и радиуса кластеров в осевом и радиальном направлениях при разных значениях числа Кнудсена. Показано, что процесс коагуляции частиц вносит существенный вклад на распределение характеристик потока в трубе. В частности, совместное влияние коагуляции и 
осаждения приводит к тому, что радиус кластеров по мере движения вдоль канала стремится к некоторому предельному значению, зависящему от определяющих параметров смеси. Обнаружено, что с увеличением числа Кнудсена характерное расстояние, на котором достигается предельный размер кластеров, сокращается, тогда как с ростом начальной концентрации частиц предельный радиус кластеров и указанное характерное расстояние увеличиваются. Установлено, что в рассматриваемых диапазонах изменения определяющих параметров распределение радиусов кластеров в радиальном направлении практически однородное. Построены аппроксимационные формулы, которые с большой точностью описывают поведение зависимостей упомянутых величин от определяющих параметров.

Полученные результаты позволяют, в частности, оценить оптимальные размеры трубы, при которых процессы осаждения и коагуляции частиц полностью завершаются.

\section{Финансирование работы}

Работа выполнена при финансовой поддержке Министерства образования и науки Республики Казахстан (грант № BR05236656).

\section{Конфликт интересов}

Авторы заявляют, что у них нет конфликта интересов.

\section{Список литературы}

[1] J. Townsend. Phil. Trans., 193, 129 (1900).

[2] P. Gormley, M. Kennedy. Proc. Roy. Irish Acad., 52A, 163 (1949).

[3] Н.А. Фукс. Механика аэрозолей (Изд. АН СССР, М., 1955) [N.A. Fuchs. The Mechanics of Aerosols (Dover, NY.,1989).]

[4] Н.А. Фукс, А.Г. Сутугин. Успехи химии, 11, 1965 (1968).

[5] C.N. Davies. J. Aerosol Sci., 4 (4), 317 (1973).

[6] E.O. Knutson. Aerosol Science and Technology, 31 (2-3), 83 (1999).

[7] Ю.Н. Самсонов. Коллоид. журнал, 75 (6), 771 (2013). [Y.N. Samsonov. Colloid J., 75 (6), 706 (2013).]

[8] Е.Р. Щукин, Н.В. Малай, З.Л. Шулиманова. Науч. ведомости БелГУ. Сер. Матем., Физ., 26, 83 (2013).

[9] B. Asgharian, F.J. Miller, O. Price, J.D. Schroeter, D.R. Einstein, R.A. Corley, T. Bentley. J. Aerosol Sci., 99, 107 (2016). DOI: https://doi.org/10.1016/j.jaerosci.2016.01.016

[10] Н.В. Малай, Е.Р. Щукин. ЖТФ, 89(4), 500 (2019). [N.V. Malai, E.R. Shchukin. Tech. Phys., 64 (4) 458 (2019).]

[11] В.И. Аникеев, Д.А. Степанов, А. Ермакова. Теоретические основы химической технологии, $45(2), 155$ (2011). [V.I. Anikeev, D.A. Stepanov, A. Ermakova. Theor. Foundations Chem. Engineer., 45 (2), 141 (2011).]

[12] П. Райст. Аэрозоли. Введение в теорию (Мир, М., 1987). [P. Reist. Introduction to Aerosol Science (A Division of Macmillan, NY., 1984).]

[13] S.E. Pratsinis, K.-S. Kim. J. Aerosol Sci., 20 (1), 101 (1989).
[14] F.E. Kruis, K.A. Kusters, S.E. Pratsinis, B. Scarlett. Aerosol Science and Technology, $19(4), 514$ (1993). DOI: $10.1080 / 02786829308959656$

[15] М.В. Тимофеева. ЖТФ, 89 (4), 491 (2019). [M.V. Timofeeva. Tech. Phys., 64 (4), 449 (2019).]

[16] S.H. Park, S.N. Rogak. Aerosol Science and Technology, 37 (12), 947 (2003).

[17] C.N. Davies. J. Aerosol Sci., 10 (2), 151 (1979).

[18] Е.С. Асмолов. Поперечная миграция малых частии в сдвиговых и нестационарных потоках. Автореферат (МГУ им. М.В. Ломоносова, М., 2015), 35 с.

[19] W.F. Phillips. Phys. Fluids, 18 (9), 1089 (1975).

[20] A.A. Lushnikov. Introduction to Aerosols. Chapt. 1 in „Aerosols - Science and Technology“ (Wiley-VCH Verlag GmbH\&Co, KGaA, Weinheim, 2010)

[21] N.A. Fuchs, I.V. Stechkina. Trans. Faraday Soc., 58, 1949 (1962).

[22] R.A. Gussman. J. Applied Meteorology, 8, 999 (1969).

[23] W.W. Szymanski, A. Majerowicz, P.E. Wagner. Aerosol Science and Technology, 11 (1), 1 (1989).

[24] P.E. Wagner, M. Kerker. J. Chem. Phys., 66, 638 (1977).

[25] S.K. Loyalka. J. Colloid Interface Sci., 57 (3), 578 (1976).

[26] M. Sitarski, J.H. Seinfeld. J. Colloid Interface Sci., 61 (2), 261 (1977).

[27] B. Nowakowski, M. Sitarski. J. Colloid Interface Sci., 83 (2), 614 (1981).

[28] В.М. Пасконов, В.И. Полежаев, Л.А. Чудов. Численное моделирование процессов тепло- и массообмена (Наука, М., 1984) 